\title{
Predictors of Loss to Follow Up of Patients Enrolled on Antiretroviral Therapy: A Retrospective Cohort Study
}

\section{Kidane Tadesse* and Fisaha Haile}

Mekelle University, College of Health Science, Department of Public Health, Mekelle, Ethiopia

*Corresponding author: Kidane Tadesse, Mekelle University, College of Health Science, Department of Public Health, Mekelle, Ethiopia, Tel: 251913505584; E-mail: kiducs98@yahoo.com

Received date: Sep 26, 2014; Accepted date: November 27, 2014; Published date: December 02, 2014

Copyright: (C) 2014 Tadesse K, et al. This is an open-access article distributed under the terms of the Creative Commons Attribution License, which permits unrestricted use, distribution, and reproduction in any medium, provided the original author and source are credited.

\begin{abstract}
Introduction: There is a growing concern about the increasing rates of Loss to Follow up (LTFU) among people who are on HIVIAIDS treatment programs. It is more common in resource-poor settings. However little is known about the time to LTFU and predictors after Antiretroviral Therapy (ART) initiation in low resource settings including Ethiopia.
\end{abstract}

Method: Retrospective cohort study was employed among a total of 520 records of patients who were enrolled on antiretroviral therapy in Aksum St. Marry hospital. Baseline patient records were extracted from electronic and paper based medical records database and analysed using Kaplan Meier survival and Cox proportional hazard model to identify the independent predictors of loss to follow up of patients on ART.

Result: of 520 patients, $51(9.8 \%)$ were loss giving a LTFU rate of 8.2 per 100 person- years. From these LTFU, $21(41 \%)$ occurred within the first Six months of ART initiation. The independent predictors of LTFU of patient were being smear positive pulmonary TB [Adj. $\mathrm{HR}(95 \% \mathrm{Cl})=(2.05(1.02,4.12)]$, male gender $[\mathrm{Adj} . \mathrm{HR}(95 \% \mathrm{Cl})=(2.73$ $(1.31,5.66)]$, regiment AZT-3TC-NVP [Adj. HR $(95 \% \mathrm{Cl})=(3.47(1.02,11.83)]$ and weight $\geq 60 \mathrm{~kg}[\mathrm{Adj}$. HR (95\% $\mathrm{Cl})=(3.47(1.02,11.83)]$.

Conclusion: Substantial magnitude of loss to follow up has been found among patients on ART which significantly affect the overall outcome of HIVIAIDS program of treatment. The independent predictors identified were TB smear positive, male gender, regiment AZT-3TC-NVP and lower weight. So, continuous and comprehensive follow up is necessary to minimize loss to follow up and optimize treatment outcome of people on ART.

Keywords: Loss to follow up; ART; Attrition, Aksum

\section{Introduction}

There is a growing concern about the increasing rates of Loss to Follow up (LTFU) in HIV programs among people already on treatment [1]. But the effectiveness of highly-active Antiretroviral Therapy (ART) could vary from region to region because of the difference in the retention of patients on ART [2]. Attrition rates in African cohort were higher than cohorts from Asian and Latin America. Reasons for the different outcomes likely include severe resource constraints in African health systems, opportunistic infections such as tuberculosis, frequently occurring in the first three months are more difficult to diagnose and treat in poorly developed health systems and a likely explanation for the higher attrition reported by African cohorts [3].

Studies from sub-Saharan Africa had shown that the cumulative incidence of attrition after 3 years of follow-up can be up to $35 \%$. A declined from $86 \%$ at 6 months to $77 \%$ by 36 months after ART initiation in sub Saharan Africa [4,5]. So, retention in care of adolescents with HIV remains a major operational challenge requiring innovation and creativity but data from low- or middle-income countries are scarce $[6,7]$.
Loss to follow-up is more common in resource-poor settings. In an Antiretroviral Treatment in lower income countries study, loss to follow-up after 1 year was above $40 \%$ in some programs, and associated with more advanced clinical disease and lower CD4 cell counts [8]. The first 6 months of ART initiation is so crucial that program managers and service providers should give due attention during this early course of treatment. Retention of patients in care remains a major challenge to the health care and program mangers [9].

However little is known about the time to attrition and predictors of LTFU after ART initiation in low resource settings including Ethiopia. So, a better understanding of the risk factors associated with attrition could be helpful to design interventions to reduce LTFU and improve clinical outcome of patients who initiate ART. Consequently, the aim of this study is to describe the attrition after ART initiation in a cohort of patients attending ART and identify the predictors of lost to follow up.

\section{Materials and Methods}

\section{Study design and setting}

A retrospective cohort study was conducted among HIV patients on ART using records between September 2006 to February 2011. The study was conducted in Aksum St. Marry hospital found in Aksum 
Page 2 of 6

town located $1067 \mathrm{Km}$ north of Addis Ababa. The hospital serves as a referral for an estimated population of 1.2 million. The Hospital starts ART service on September 2005.

\section{Study population and sampling techniques}

The study population was all ART enrolled patient within the specified study period. The total sample size was calculated using stata stpower cox and found to be 520 patient records. Regarding the sampling technique, record of study participants have been filtered first from the database according to their entry time to the follow up, next patients have been filtered using age and eligibility criteria then we give a unique number for the remaining records and select each record for our study using systematic random sampling. The outcome variable was time to event (LTFU) of patients on ART. Data about LTFU was collected from patents' charts.

\section{Data collection technique and data quality control}

A standard checklist was used for recording information extracted from patient cards. This form is developed using the National standardized ART entry and follow up form employed by the ART clinic. The CD4 count laboratory results recorded before starting ART were used as a base line values. If there is no pre-treatment laboratory test, however, results obtained within one month of ART initiation were considered as baseline values. The checklist consists of variables that are recorded at ART initiation (baseline) such as Sex, Age, Marital status, Educational status, CD4 cell, Baseline weight (kg), Hemoglobin $(\mathrm{g} / \mathrm{dL})$, WHO clinical stage, TB smear, Original ART regimen, CPT initiation and presence of Opportunistic Infections (OIs). Four experienced ART nurses who were trained on comprehensive HIV care and involved in patient follow ups collected the data and data collection was supervised by trained supervisors. During the data collection process the checklist was checked for their completeness, consistency and accuracy by the principal investigator every day. The data were entered, cleaned and analysed using Stata version 11.1.

\section{Data management and analysis}

Exploratory data analysis was carried out to check the levels of missing values, presence of influential outliers, multi-colinearity, normality and proportionality of hazards over time. The proportions of the outcome were reported as alive, dead, loss to follow up and transfer out. The median follow up time of the cohort was also computed at 12, 24 and 60 months. Person-months of follow up were calculated by assessing the date of enrolment for ART and LTFU or censoring. Kaplan Meier (KM) was used to estimate rates at particular times and generate a survival curve. Log Rank test was used to test the equality of survival probabilities and compared across the different groups of covariates. The overall survival function and separate estimates for the stratum of covariates were considered as statistically significant at p-value 0.05 in the Log- rank test. Hazard Ratios (HR) with $95 \%$ confidence intervals were used as effect measures. Multivariable Cox proportional hazards regression was used to model the contribution of baseline demographic characteristics and clinical characteristics on LTFU of adult persons on ART for variables with $p$, 0.05 in bivariate analysis. This method has been used for the estimation of Adjusted Hazard Ratios (AHR) at 95\% confidence intervals for the LTFU rate among patients on ART for the covariates at their ART initiation.

\section{Ethical statement}

After assurance of anonymity, verbal consent for those who can't read and write and written Consent for those who can read and write was obtained from all patients attending ART to store and use the information obtained during their first visit and subsequent follow ups. All information collected from patients cards were kept strictly confidential and names of patients on ART were not included in the data abstraction form. All the documentations and strategies used to maintain the Ethical principles has been provided to the Health research Ethics review committee of Mekelle University College of Health Science (HRERC) and clearance was secured after evaluation of the study protocol. Finally written support letter was obtained from Mekelle University and Tigray Regional Health Bureau to Aksum St. Merry hospital. All patient data was accessed after the purpose of the research and ethical principles had discussed with the hospital's authority.

\section{Result}

\section{Cohort characteristics}

A total of 520 patient records enrolled on ART were included in this study. The mean age and standard deviation of patients was $(35.4 \pm$ 8.4) years. More than half (57\%) of patients had no or primary education. Majority (60\%) of patients were married. Most $(72.8 \%)$ of patients were alive at the end of study period, $9.8 \%$ were loss to follow up, $8.5 \%$ had transferred out to other health facilities and $8.9 \%$ patients were died (Table 1). The median follow up time for the cohort was 32 months with Interquartile range of $(22,45)$ months. Around $78 \%$ of the participants have CD4 cell count of less than 200 cells $/ \mathrm{ml}$ at baseline. Majority (78\%) of the patients were under WHO stage III or IV. As we observe from Table 2 around $43 \%$ of patients were having lower haemoglobin (anemia) at baseline. The mean level of haemoglobin with standard deviation was $(12.86 \pm 1.99) \mathrm{mg} / \mathrm{dl}$. Thirteen per cent of the patients have smeared positive pulmonary TB at baseline (Table 2).

\begin{tabular}{|l|l|l|l|}
\hline Characteristics & Number & $\%$ & Event (LTFU) \\
\hline Age & 35 & 6.73 & 3 \\
\hline $15-24$ years & 210 & 40.38 & 25 \\
\hline $25-34$ years & 185 & 35.58 & 16 \\
\hline $35-44$ years & 90 & 17.31 & 7 \\
\hline$\geq 45$ & \multicolumn{5}{|l|}{} \\
\hline Gender & 225 & 43.27 & 33 \\
\hline Male & 295 & 56.73 & 18 \\
\hline Female & \multicolumn{5}{|l|}{} \\
\hline Marital status & 101 & 19.42 & 13 \\
\hline Never Married & 314 & 60.38 & 24 \\
\hline Married & 12 & 2.31 & 1 \\
\hline Separated & 43 & 8.27 & 6 \\
\hline Divorced & 50 & 9.62 & 7 \\
\hline Widowed & &
\end{tabular}


Page 3 of 6

\begin{tabular}{|l|l|l|l|}
\hline Level of Education & 117 & 22.50 & 11 \\
\hline No education & 179 & 34.42 & 16 \\
\hline Primary & 180 & 34.62 & 18 \\
\hline Secondary & 44 & 8.46 & 6 \\
\hline Tertiary & \multicolumn{5}{|l|}{} \\
\hline Religion & 51 & 9.81 & 6 \\
\hline Muslim & 468 & 90.00 & 45 \\
\hline Orthodox & 1 & 0.19 & 0 \\
\hline Protestant & 411 & 79.04 & 42 \\
\hline Dependent children & 109 & 20.96 & 9 \\
\hline Yes & \multicolumn{5}{|l|}{} \\
\hline No &
\end{tabular}

Table 1: Base line patients' characteristics, 2006-2011, Aksum St. Merry Hospital, Northern Ethiopia

\begin{tabular}{|c|c|c|c|}
\hline Characteristics & Number & $\%$ & Event \\
\hline \multicolumn{4}{|l|}{ TB smear } \\
\hline Not determined & 103 & 19.81 & 10 \\
\hline Negative & 346 & 66.54 & 28 \\
\hline Positive & 71 & 13.65 & 13 \\
\hline \multicolumn{4}{|l|}{ Original Regimen } \\
\hline d4t (30)-3TC-NVP & 161 & 30.96 & 15 \\
\hline d4t (40)-3TC-NVP & 101 & 19.42 & 8 \\
\hline d4t (30)-3TC-EFV & 139 & 26.73 & 8 \\
\hline d4t (40)-3TC-EFV & 93 & 17.88 & 16 \\
\hline AZT-3TC-NVP & 21 & 4.04 & 4 \\
\hline AZT-3TC-EFV & 5 & 0.96 & 0 \\
\hline \multicolumn{4}{|c|}{ CD4 count ( cells $/ \mathrm{mm}^{3}$ ) } \\
\hline$\leq 50$ & 96 & 18.46 & 14 \\
\hline $51-100$ & 101 & 19.42 & 11 \\
\hline $101-200$ & 207 & 39.81 & 14 \\
\hline$\geq 201$ & 116 & 22.31 & 12 \\
\hline \multicolumn{4}{|l|}{ Cotimoxazole } \\
\hline Yes & 429 & 82.50 & 41 \\
\hline No & 91 & 17.50 & 10 \\
\hline \multicolumn{4}{|l|}{ Weight (Kg) } \\
\hline$\leq 40$ & 97 & 18.65 & 12 \\
\hline $40-50$ & 225 & 43.27 & 23 \\
\hline
\end{tabular}

\begin{tabular}{|l|l|l|l|}
\hline $50-60$ & 147 & 28.27 & 13 \\
\hline$>60$ & 51 & 9.81 & 3 \\
\hline Functional status & 265 & 50.96 & 17 \\
\hline Working & 214 & 41.15 & 30 \\
\hline Ambulatory & 41 & 7.88 & 4 \\
\hline Bedridden & \multicolumn{5}{|l|}{} \\
\hline WHO stage & 33 & 6.35 & 3 \\
\hline Stage I & 83 & 15.96 & 3 \\
\hline Stage II & 336 & 64.62 & 39 \\
\hline Stage III & 68 & 13.08 & 6 \\
\hline Stage IV & \multicolumn{5}{|l|}{} \\
\hline Hemoglobin (g/dl) & 223 & 42.88 & 14 \\
\hline$\leq 11$ & 297 & 57.12 & 37 \\
\hline$>11$ & &
\end{tabular}

Table 2: Base line Patients' Clinical characteristics, 2006-2011, Aksum St. Merry Hospital.

\section{LTFU and its Predictors}

A total of 520 patients contributed to 1, 400 person-years of follow up and 51 patients loss giving a LTFU rate of 8.2 per 100 person- years. Of those LTFU, 21(41\%) occurred within the first Six months of ART initiation. On the other hand, the median survival time to LTFU was 14 months with Interquartile range of 8 to 27 months. Lost to followup increases as the time spent on ART treatment increases (Table 3). The cumulative stay of this cohort shows decrement over time $95 \%$ to $85 \%$ at 12 and 60 months. In multivariate Cox regression, patients diagnosed with TB had a 2-fold higher risk of LTFU (Adjusted Hazard Ratio (AHR) $=2.05,95 \%$ CI: 1.02, 4.12) than individuals who were TB negative. Being male have almost 3 fold higher risk of loss $(A H R=2.7$, $95 \%$ CI: $1.31,5.66$ ). Likewise, patients with weight above $60 \mathrm{~kg}$ have $76 \%$ lower risk of being loss to follow up than their counter parts(AHR $=0.24,95 \%$ CI: $0.06,0.96)$ and those with at regiment AZT-3TC-NVP have around 3.5 times increased risk of loss to follow up than those individual who were using d4t (30)-3TC-NVP (AHR=3.47, 95\% CI :1.02,11.83) (Table 4).

\section{Discussion}

This study has assessed the level of LTFU and its associated factors in ART enrolled adults. Accordingly, there were a total of $51(9.8 \%)$ patients' lost from their ART treatment giving a LTFU rate of 8.2 per 100 person-years. The same study conducted in Ethiopia reported that $15 \%$ of LTFU has occurred with in the first six months [9]. Similarly, another study conducted in French hospitals databases of HIVinfected patients found $8.5 \%$ LTFU and the incidence rate of LTFU was estimated to be 3.5 per 100 person-years, a much lower estimate than this study [10]. But another study conducted in India $h$ found an incidence rate of LTFU 7.1 per 100 person-years [11-13]. Findings of 
Page 4 of 6

this study and all the other studies show almost similar and substantial magnitude of LTFU from ART treatment. However the incidence of LTFU in this study is higher than the incidence of lost to follow up in the French study. This may be explained by the variations in the study settings between the two studies.

\begin{tabular}{|l|l|l|}
\hline Month of Follow up & Cumulative Failure & $\mathbf{9 5 \%} \mathbf{~ C l}$ \\
\hline 12 & 0.047 & $0.032,0.070$ \\
\hline 24 & 0.076 & $0.056,0.104$ \\
\hline 36 & 0.100 & $0.075,0.133$ \\
\hline 48 & 0.131 & $0.099,0.174$ \\
\hline 60 & 0.141 & $0.105,0.187$ \\
\hline
\end{tabular}

Table 3: Cumulative failure (lost to follow up) of patients on ART, September 2006 to August 2011, Aksum St. Merry Hospital, Northern Ethiopia.

\begin{tabular}{|c|c|c|c|}
\hline Variables & AHR & P-value & $95 \% \mathrm{Cl}$ \\
\hline \multicolumn{4}{|l|}{ Sex } \\
\hline Female & Reference & & \\
\hline Male & 2.73 & 0.007 & $1.31,5.66$ \\
\hline \multicolumn{4}{|l|}{ TB-smear } \\
\hline Negative & Reference & & \\
\hline Positive & 2.05 & 0.04 & $1.02,4.12$ \\
\hline Undetermined & 1.45 & 0.32 & $0.64,4.44$ \\
\hline \multicolumn{4}{|l|}{ Regimen } \\
\hline d4t (30)-3TC-NVP & Reference & & \\
\hline d4t (40)-3TC-NVP & 0.63 & 0.34 & $0.25,1.63$ \\
\hline d4t (30)-3TC-EFV & 0.83 & 0.69 & $0.34,2.04$ \\
\hline d4t (40)-3TC-EFV & 1.8 & 0.18 & $0.75,4.32$ \\
\hline AZT-3TC-NVP & 3.47 & 0.04 & $1.02,11.83$ \\
\hline AZT-3TC-EFV & 1.57 & 0.23 & $0.85,6.46$ \\
\hline \multicolumn{4}{|l|}{ Functional status } \\
\hline Working & Reference & & \\
\hline Ambulatory & 1.66 & 0.11 & $0.88,3.12$ \\
\hline Bedridden & 1.03 & 0.96 & $0.33,3.24$ \\
\hline \multicolumn{4}{|l|}{ CD4cat1 (cells $/ \mathrm{mm}^{3}$ ) } \\
\hline$<50$ & Reference & & \\
\hline $50-100$ & 0.84 & 0.67 & $0.38,1.87$ \\
\hline $101-200$ & 0.71 & 0.4 & $0.33,1.56$ \\
\hline$>200$ & 1.15 & 0.74 & $0.49,2.68$ \\
\hline
\end{tabular}

\begin{tabular}{|l|l|l|l|}
\hline$\leq 40$ & Reference & & \\
\hline $40-50$ & 0.74 & 0.44 & $0.34,1.58$ \\
\hline $50-60$ & 0.6 & 0.26 & $0.25,1.46$ \\
\hline$>60$ & 0.24 & $0.046^{*}$ & $0.06,0.96$ \\
\hline Hemoglobin $(g / d l)$ & Reference & & \\
\hline Anemic & 0.49 & 0.054 & $1.02,4.12$ \\
\hline Normal & \multicolumn{5}{|l}{} \\
\hline
\end{tabular}

Table 4: Multivariate Cox regression of baseline predictors, 20062011, Aksum St. Merry Hospital, Northern Ethiopia. D4T: Stavudine; EFV: Efavirenz; AZT: Zidovudine; NVP: Nevirapine; 3TC: Lamiduvine; AHR: Adjusted Hazard Ratio; CI: Confidence Interval.

Of these 51 LTFU, 21(41\%) of them have occurred within the first Six months of ART initiation. Similar studies done in different areas have shown that there was a higher LTFU rate at early months of ART initiation [11,12]. Rates of loss to follow-up declined in Zambia from 211.6 per 1,000 person-years in the first 6 months of ART to 53.5 per 1,000 person-years thereafter 26 .

Males have higher risk of being loss from the ART service i.e. a twofold high risk of being LTFU compared to females. Different studies done in developing countries in Africa also showed that there is higher risk of LTFU in males than females [14-18]. Similarly, according to a study conducted in India, there was $37 \%$ less likely risk of being LTFU for females [13]. This could explain by the reason that men travel for work particularly in professions of truck drivers, fishermen, and migrant agricultural workers which keeps them away from their home likewise their medication $[19,20]$.

In this study LTFU rate was associated with baseline body weight of patients. It was found that patients with weight above $60 \mathrm{~kg}$ have $76 \%$ lower risk of being loss to follow up than those who have weight below $40 \mathrm{~kg}$ at baseline. This could be due to the fact that patients with normal weight would like to maintain their good wellbeing (i.e. they don't want to lose) by taking ART so that they stay longer in the program. According to study conducted in South Africa, Patients universally reported, that they had experienced important, even extraordinary health benefits from taking ART and recognized that stopping ART would lead to illness. Weight gain and improved mobility were commonly noted [21].

According to findings from this study, the baseline ART regimen of patients was found to be one of the independent predictors of loss to follow up. Those with at regiment AZT-3TC-NVP have around 3.5 times higher risk of loss to follow up than those individual who were using $1 \mathrm{~d} 4 \mathrm{t}$ (30)-3TC-NVP. A ten year Nigerian study indicates type of regimen as one of the risk factors and indicate that greater percentage (43.2\%) of AZT-3TC-NVP have adverse side effects [22]. Additionally, a study conducted in central and southern Ethiopia reported that there was a significant difference on the level of adherence of HIV infected patients to different ART regimes [23]. Consequently, this difference in adherence will have an effect on the level of loss to follow up i.e. those who have lower adherence might be at higher risk of being loss. Trotta et al. investigated which regimens were associated with the poorest rates of adherence and found that protease inhibitor (PI)treated patients were the poorest adherers [24]. 
Page 5 of 6

Patients who were TB positive at baseline had a 2 -fold higher risk of LTFU than individuals who were TB negative. There is always overlapping toxicity in using any multidrug therapy. Consequently, adverse reactions to anti tuberculosis drugs are common among patients with HIV related tuberculosis. Adverse reactions to antiretroviral drugs are also common, and there are significant overlaps in the toxicity profiles of the first-line anti tuberculosis and antiretroviral drugs [25].

This study might suffer from limitations that the retrospective cohort nature of the study and it also limits our ability to gather data about factors that may influence the risk of lost to follow up, like information about patient behaviors, family supports, cares given at family level, adherence to treatment, etc.

In conclusion, substantial magnitude of lost to follow up has been observed which may substantially affect program outcome. Additionally there was higher rate of LTFU in the first six months after ART enrollment. The major independent baseline patient characteristics which affect loss to follow up were: TB status, male gender, and weight and ART regiment. So, continuous and comprehensive follow up is necessary to minimize loss to follow up and optimize treatment outcome of people on ART with special emphasis male, pulmonary positive TB patients especially at the early periods of ART initiation in Ethiopia and other similar settings of developing countries.

\section{Authors' contributions}

Kidane Tadesse: Involved in proposal writing, designing, and recruitment and training of Supervisors and data collectors, analysis and write-up and in all stages of the project implementation. He did most of the analysis and write up of the paper. Fisaha Haile: Involved in proposal writing, designing, and recruitment and training of Supervisors and data collectors, analysis and write-up and in all stages of the project implementation.

\section{Acknowledgement}

The Authors are grateful to the Mekelle University, College of Health Sciences for sponsoring this research project. We also extend sincere appreciation to all health workers of Axum Hospital, who helped us during data collection. Last but not least, we were grateful to the data collectors and supervisors for carefully undertaking of their tasks.

\section{References}

1. Ahmed I Gugsa ST, Lemma S, Demissie M (2013) Predictors of loss to follow-up before HIV treatment initiation in Northwest Ethiopia: a case control study. BMC Public Health 13: 867.

2. Abebe N, Alemu K, Asfaw T, Alemu A (2014) Predictors of mortality among HIV positive adults on antiretroviral therapy in Debremarkos Referral Hospital, Northwest Ethiopia. Journal of AIDS and HIV Research 6: 19-27.

3. Renaud-Théry F, Duncombe C, Kerr S (2014) Thierry S, PerriënsJ: Adult antiretroviral therapy in resource limited settings: a systematic review of first-line failure and attrition rates.

4. Alvarez-Uria GNaik PK, Pakam R, Midde M (2013) Factors associated with attrition, mortality, and loss to follow up after antiretroviral therapy initiation: data from an HIV cohort study in India. Glob Health Action 6: 21682.

5. Mutasa-Apollo T, Shiraishi RW, Takarinda KC, Dzangare J, Mugurungi O, Murungu J, et al. (2014) Patient Retention, Clinical Outcomes and
Attrition-Associated Factors of HIV-Infected Patients Enrolled in Zimbabwe's National Antiretroviral Therapy Programme,2007-2010. PLoS One 9: e86305.

6. Alvarez-Uria G, Midde M, Pakam R, Naik PK (2013) Predictors of attrition in patients ineligible for antiretroviral therapy after being diagnosed with HIV: data from an HIV cohort study in India. Biomed Res Int 2013: 858023.

7. William M, Rebecca L, Paola C, Martina P, Charles N, et al. (2013) Attrition and loss to follow-up Among Children and Adolescents in a Community Home-Based Care HIV Programme in Uganda. Pediat Therapeut 3:5.

8. Schöni-Affolter F, Keiser O, Mwango A, Stringer J, Ledergerber B, et al. (2011) Estimating Loss to Follow-Up in HIV-Infected Patients on Antiretroviral Therapy: The Effect of the Competing Risk of Death in Zambia and Switzerland. PLoS One 6: e27919.

9. Assefa Y, Kiflie A, Tesfaye D, Mariam DH, Kloos H, et al. (2011) Outcomes of antiretroviral treatment program in Ethiopia: Retention of patients in care is a major challenge and varies across health facilities. BMC Health Serv Res 18: 11-81.

10. Lanoy E, Mary-Krause M, Tattevin P, Dray-Spira R, Duvivier C, et al. (2006) Predictors identified for losses to follow-up among HIVseropositive patients. J Clin Epidemiol 59: 829-835.

11. Arici C, Ripamonti D, Maggiolo F, Rizzi M, Finazzi MG, et al. (2002) Factors associated with the failure of HIV-positive persons to return for scheduled medical visits. HIV Clin Trials 3: 52-57.

12. Ndiaye B, Ould-Kaci K, Salleron J, Bataille P, Bonnevie F, et al. (2009) Incidence rate and risk factors for loss to follow-up in HIV-infected patients from five French clinical centres in Northern France - January 1997 to December 2006. Antivir Ther 14: 567-575.

13. Alvarez-Uria G, Pakam R, Midde M, Naik P (2013) Factors associated with attrition, mortality, and loss to follow up after antiretroviral therapy initiation: data from an HIV cohort study in India. Glob Health Action 6: 21682.

14. Maskew M, MacPhail P, Menezes C, Rubel D (2007) Lost to follow up: contributing factors and challenges in South African patients on antiretroviral therapy. S Afr Med J 97: 853-857.

15. Ochieng-Ooko V Ochieng D, Sidle JE, Holdsworth M, Wools-Kaloustian $\mathrm{K}$, et al. (2010) Influence of gender on loss to follow-up in a large HIV treatment programme in western Kenya. Bull World Health Organ 88: 681-688.

16. Makombe SD, Jahn A, Tweya H, Chuka S, Yu JK, et al. (2007) A national survey of teachers on antiretroviral therapy in Malawi: access, retention in therapy and survival. PLoS One 2: e620.

17. Ekouevi DK, Balestre E, Ba-Gomis FO, Eholie SP, Maiga M, et al. (2010) Low retention of HIV-infected patients on antiretroviral therapy in 11 clinical centres in West Africa. Trop Med Int Health 15: 34-42.

18. Mugglin C, Estill J, Wandeler G, Bender N, Egger M, et al (2012) Loss to programme between HIV diagnosis and initiation of antiretroviral therapy in sub-Saharan Africa: systematic review and meta-analysis. Tropical Medicine and International Health, Blackwell Publishing Ltd.

19. Lagarde E, Schim van der Loeff M, Enel C, Holmgren B, Dray-Spira R, et al. (2003) Mobility and the spread of human immunodeficiency virus into rural areas of West Africa. Int J Epidemiol 32: 744-752.

20. Hunt CW (1989) Migrant labor and sexually transmitted disease: AIDS in Africa. J Health Soc Behav 30: 353-373.

21. Miller MC, Ketlhapile M, Rybasack-Smith H, Rosen S (2010) Why are anti-retroviral treatment patients loss to follow up? A qualitative study from South Africa. Tropical Medicine \& International Health 15: 48-54.

22. Reginald AO, Haruna MM, Sani GB, Eric T, Adebola OT, et a. (2012) Reactions Associated with Antiretroviral Regimens in Adult Patients of a University Teaching Hospital HIV Program in Zaria, Northern Nigeria: An Observational Cohort Study. J Antivir Antiretrovir 4: 006-013.

23. Beyene KA, Gedif T, Gebre-Mariam T, Engidawork E (2009) Highly active antiretroviral therapy adherence and its determinants in selected hospitals from south and central Ethiopia. Pharmacoepidemiol Drug Saf 18: $1007-1015$. 
Citation: Tadesse K, Haile F (2014) Predictors of Loss to Follow Up of Patients Enrolled on Antiretroviral Therapy: A Retrospective Cohort Study. J AIDS Clin Res 5: 393. doi:10.4172/2155-6113.1000393

Page 6 of 6

24. Trotta MP, Ammassari A, Cozzi-Lepri A, Zaccarelli M, Castelli F, et al. (2003) Adherence to highly active antiretroviral therapy is better in patients receiving non-nucleoside reverse transcriptase inhibitor containing regimens than in those receiving protease inhibitorcontaining regimens. AIDS 17: 1099-1102.
25. Perriens JH, St. Louis ME, Mukadi YB, Brown C, Prignot J, et al. (1995) Pulmonary tuberculosis in HIV-infected patients in Zaire: a controlled trial of treatment for either 6 or 12 months. N Engl J Med 332: 779-784. 